Herz und Psyche gehen Hand in Hand

\title{
Sichere antidepressive Therapie bei Patienten mit somatischen Begleiterkrankungen
}

— Zwischen Herz und Psyche besteht ein bidirektionaler Zusammenhang: So sind Postinfarkt-Patienten stark gefährdet, auch an einer Depression zu erkranken, die dann ihrerseits wiederum Reinfarkt-Risiko und Mortalität erhöht, berichtete Priv.-Doz. Dr. Kai Kahl, Hannover. Zudem ist die Depression als kardiovaskulärer Risikofaktor anzusehen, der die Entwicklung von Hochdruck, Atherosklerose und Diabetes begünstigt. Kahl bezeichnete die Depression als Psychosomatose: „Die Depression findet nicht nur im Kopf statt, sondern betrifft endokrinologische und immunologische Systeme, die metabolische Veränderungen im Organismus befeuern."

Als schwere Erkrankung sollte die Depression bereits ab der ersten Episode suffizient behandelt werden, um eine Chronifizierung zu verhindern. Das erfordert laut Kahl häufig eine Langzeittherapie. Damit Patienten therapeutisch bei der Stange bleiben, sollten nicht nur effektive, sondern auch nebenwirkungsarme Antidepressiva gewählt werden, die z. B. nicht mit gleichzeitig einzunehmenden Medikamenten wie internistischen Arzneimitteln interagieren. Auch sollten kardiovaskuläre Risikofaktoren nicht noch weiter negativ beeinflusst werden. Kahl wies auf SSRI hin, die langfristig zur Gewichtszunahme führen können und das Risikoprofil weiter in Richtung metabolisches Syndrom verschieben.

Als günstig bezeichnete er in dieser Hinsicht das melatonerge Antidepressivum Agomelatin (Valdoxan ${ }^{\circledR}$ ), das gewichtsneutral ist und über eine gute kardiovaskuläre Verträglichkeit verfügt. Für die hohe Effektivität und gute Verträglichkeit der Substanz im klinischen Alltag spricht die nicht interventionelle Studie VIVALDI-PRAXIS. Sie umfasste 1070 Patienten mit einer mittelschweren bis schweren Depression, davon drei Viertel mit somatischen Begleiterkrankungen, die in internistischen und allgemeinmedizinischen Praxen behandelt wur- den, berichtete Prof. Gerd Laux, Wasserburg. Innerhalb von zwölf Wochen hatten $78,4 \%$ der Patienten unter Agomelatin respondiert; fast zwei Drittel $(63,5 \%)$ erreichten eine Remission. Vielfach wurde bereits ein frühes Ansprechen in der zweiten Studienwoche $(61,7 \%$ sog. Early Improver) beobachtet.

Laux wies auf den starken positiven Effekt von Agomelatin auf den Schlaf-WachRhythmus hin: Ein- und Durchschlafstörungen sowie Tagesmüdigkeit bildeten sich "eindrucksvoll" zurück. Nebenwirkungen wurden nur bei 2,5\% der Patienten beobachtet, obwohl über $60 \%$ der Patienten Begleitmedikamente erhielten. Laux bescheinigt Agomelatin daher eine "gute Verträglichkeit in Komedikation mit internistischen Substanzen".

- Katharina Arnheim

Quelle: Symposium „Herz und Seele - eine verhängnisvolle Beziehung", DGIM-Kongress, Wiesbaden, April 2012 (Veranstalter: Servier)

\section{Ärzte mit innovativen Ideen gesucht}

Innovationen bringen das Gesundheitswesen voran. Auch viele niedergelassene Ärzte entwickeln in ihren Praxen innovative Ideen, um die Versorgung ihrer Patienten zu verbessern, den Aufwand in den Praxen angesichts eines wachsenden $\mathrm{Pa}$ tientenzustroms zu begrenzen und den wirtschaftlichen Ertrag der Praxis zu steigern. Innovationen helfen Praxen auch, im Wettbewerb mit größeren Zentren wie MVZ zu bestehen. Doch nicht immer setzen sich gute neue Ideen durch - einfach weil sie nicht bekannt sind.

Die Fachverlagsgruppe Springer Medizin und das Biopharmaunternehmen UCB wollen einen Beitrag dazu leisten, dass innovative Ärzte zu Vorbildern werden. Deshalb schreiben sie zum zweiten Mal den Wettbewerb "Die innovative Arztpraxis" aus. Gewinnerin des Innova- tionspreises 2011 war die hausärztliche Internistin Irmgard Landgraf, Berlin. Sie arbeitet eng mit einem Pflegeheim zusammen und hat über ihren Praxisrechner oder auch per Laptop Zugriff auf die Patientendaten des Heims. Das ermöglicht eine bessere Patientenversorgung.

„Auch für den Innovationspreis 2012 suchen wir wieder kreative Ärzte, die die Herausforderung annehmen, eine innovative Medizin zu betreiben und die Versorgung zu verbessern", sagt Steffen Fritzsche, Leiter Unternehmenskommunikation bei UCB. Gesucht werden z. B. Ideen

- bei Praxisöffnungszeiten und Terminvergabe,

- in modernen Kooperationsmodellen mit Kollegen, Apotheken, Fitness-Studios, Altenheimen, der Industrie etc.
- in der Nutzung von Tablet-PCs für die Patientenaufklärung und/oder fürs (IGeL-)Marketing,

- in der Kommunikation des Angebots der Praxis (Werbung, Flyer etc.),

- in der Nutzung von Kommunikationsmitteln bei Patientenkontakten (Telefon, Fax, E-Mail, Social Media etc.),

- in der internen Praxisorganisation, etwa im Fehlermanagement oder Qualitätsmanagement.

Sie können sich bis zum 31. Oktober 2012 unter www.aerztezeitung.de/innovationspreis2012 bewerben. Drei iPads sind zu gewinnen. Die Preisverleihung findet am 24. November 2012 beim Bundeskongress Privatmedizin in Köln statt. Bei Rückfragen wenden Sie sich an: innovation@aerztezeitung.de (Stichwort: Innovationspreis). 